Vulnerabilidades urbanas en los países andinos

(Bolivia, Ecuador, Perú)

\title{
Los mecanismos de transmisión de vulnerabilidad en el medio urbano. Primeros elementos de reflexión
}

Les mécanismes de transmission de vulnérabilité en milieu urbain. Premiers

éléments de réflexion

Vulnerability transmission mechanisms in urban environment. First thoughts

Pascale Metzger y Robert D'Ercole

\section{(2) OpenEdition}

\section{Journals}

Edición electrónica

URL: http://journals.openedition.org/bifea/2570

DOI: $10.4000 /$ bifea. 2570

ISSN: 2076-5827

\section{Editor}

Institut Français d'Études Andines

Edición impresa

Fecha de publicación: 1 diciembre 2009

Paginación: 917-936

ISSN: 0303-7495

\section{Referencia electrónica}

Pascale Metzger y Robert D'Ercole, « Los mecanismos de transmisión de vulnerabilidad en el medio urbano. Primeros elementos de reflexión », Bulletin de l'Institut français d'études andines [En línea], 38

(3) | 2009, Publicado el 01 junio 2010, consultado el 10 diciembre 2020. URL : http:// journals.openedition.org/bifea/2570; DOI : https://doi.org/10.4000/bifea.2570

\section{(c)}

Les contenus du Bulletin de l'Institut français d'études andines sont mis à disposition selon les termes de la licence Creative Commons Attribution - Pas d'Utilisation Commerciale - Pas de Modification 4.0 International. 


\title{
Los mecanismos de transmisión de vulnerabilidad en el medio urbano. Primeros elementos de reflexión
}

\author{
Pascale Metzger* \\ Robert D'Ercole*
}

\begin{abstract}
Resumen
La creciente complejidad de las sociedades y las ciudades se traduce en interdependencias cada vez más marcadas entre redes y territorios, que acentúan su vulnerabilidad. Partiendo de una concepción del riesgo basada en los elementos esenciales de un territorio y su vulnerabilidad, se plantea la hipótesis de que los efectos de propagación del riesgo son interpretables mediante la noción de transmisión de vulnerabilidad. En efecto esta se efectúa a través de las relaciones cada vez más numerosas y más complejas que unen a los elementos esenciales entre sí y al territorio con tales elementos. Se trata, en este texto, de una primera tentativa, apoyada en ejemplos, de formalizar la noción de transmisión de vulnerabilidad.
\end{abstract}

Palabras clave: vulnerabilidad, elementos esenciales, ciudad, riesgo, complejidad, red, territorio, transmisión de vulnerabilidad, efectos en cadena

\section{Les mécanismes de transmission de vulnérabilité en milieu urbain. Premiers éléments de réflexion}

\section{Résumé}

La complexité croissante des sociétés et des villes se traduit par des interdépendances de plus en plus marquées entre réseaux et entre territoires qui renforcent leur vulnérabilité. En s'appuyant sur une conception du risque qui repose sur les enjeux majeurs d'un territoire et leur vulnérabilité, nous posons

* Institut de Recherche pour le Développement (IRD), UR 029, programa Pacivur. Calle Teruel 357, Miraflores, Casilla 18-1209, Lima 18, Perú. E-mails:

pascale.metzger@ird.fr

robert.dercole@ird.fr 
I'hypothèse que les effets de propagation du risque sont interprétables par la notion de transmission de vulnérabilité. En effet, la vulnérabilité se transmet via les relations de plus en plus nombreuses et de plus en plus complexes qui unissent les enjeux entre eux et ces enjeux avec le territoire. II s'agit, dans ce texte, d'une première tentative, appuyée sur des exemples, de formalisation de la notion de transmission de vulnérabilité.

Mots clés : vulnérabilité, enjeux, ville, risque, complexité, réseau, territoire, transmission de vulnérabilité, effets en chaîne

\title{
Vulnerability transmission mechanisms in urban environment. First thoughts
}

\begin{abstract}
The increasing complexity of societies and cities involves increasing interdependences between networks and between territories that reinforce their vulnerability. Based on a conception of risk based on the territorial strategic elements and their vulnerability, we explore the hypothesis that these effects of risk propagation can be construed as the concept of vulnerability transmission. Indeed, vulnerability is transmitted through an increasing number of relations of increasing complexity that links the strategic elements between them and with the territory. This text is a first attempt, based on examples, to formalize the concept of vulnerability transmission.
\end{abstract}

Key words: Vulnerability, challenges, city, risk, complexity, network, territory, vulnerability transmission, chain effects

\section{INTRODUCCIÓN}

La producción de riesgos por parte de la propia sociedad es una característica de la civilización posindustrial moderna (Beck, 2001). Esta última ampara unas amenazas globales en las que las situaciones de exposición al riesgo están cada vez más alejadas de las fuentes de peligro y son cada vez menos controlables. En directa filiación con esta afirmación, el generalizado funcionamiento en redes de los territorios, las ciudades, las sociedades, las economías, propicia los denominados nuevos riesgos, de difícil identificación y manejo (Godard et al., 2002). Este funcionamiento sistémico global se apoya ampliamente en la existencia de grandes redes vitales que garantizan la circulación y la distribución de bienes y servicios materiales o inmateriales cada vez más indispensables para el funcionamiento de las sociedades y los territorios. Se trata concretamente de las infraestructuras viales, de agua y de electricidad, aunque también de las redes de telecomunicaciones e informáticas. De manera contigua, estas redes crecen sin freno y sus interconexiones se hacen cada vez más estrechas (Egan, 2007), lo que aumenta su complejidad (Morin, 1990) y hace por tanto ilusoria la comprensión aislada del funcionamiento de una red, y con mayor razón la de un territorio. Por tal motivo, entender los riesgos en un territorio implica necesariamente comprender el territorio en su conjunto. En efecto, la demultiplicación de las redes 
y sus crecientes interconexiones, la dependencia, cada vez más marcada e incluso total, de las sociedades y los territorios frente a las redes, y la interdependencia de estos últimos complican en extremo el funcionamiento de un territorio y la identificación de las interdependencias espaciales y funcionales internas y externas que lo organizan. Al mismo tiempo acrecientan su vulnerabilidad.

A nivel urbano, el funcionamiento sistémico de las ciudades se acompaña de un doble movimiento espacial y funcional de construcción de polaridades y de marcadas especializaciones territoriales, consideradas como una evolución mayor de los conjuntos urbanos (Levy \& Lussault, 2003). Las diferentes porciones del territorio que componen la ciudad deben, para funcionar, estar conectadas entre sí y a las diferentes redes, lo que justamente va a reforzar su vulnerabilidad. En efecto, en el campo de los riesgos, estas interconexiones producen efectos más o menos inesperados, más o menos lejanos y previsibles en lo que se puede denominar una forma de «propagación de los riesgos», a escala tanto urbana como regional, nacional e incluso planetaria (Egan, 2007). Por ello, en la problemática de los riesgos, el papel de esas grandes redes vitales en el desencadenamiento o la amplificación de eventos perjudiciales es casi siempre señalado como una cuestión crucial.

«A menudo la red actúa como un medio físico de propagación del siniestro que alcanza entonces a un mayor número de víctimas, más rápidamente y de manera ubicua» (Michel-Kerjan, 1999).

Se puede entonces considerar que las redes desempeñan un papel mayor en la «propagación del riesgo» y en la construcción de la vulnerabilidad de las sociedades y los territorios. Tales grandes redes vitales ofrecen por tanto, al menos hipotéticamente, la posibilidad de trazar la trayectoria de la propagación del riesgo. Esto se puede realizar basándose por una parte en la identificación de las interconexiones e interdependencias entre lugares y entre redes, y por otra en la búsqueda de los mecanismos de producción de los «efectos secundarios» más o menos lejanos y discontinuos en relación con una situación de riesgo identificada. Sin embargo, estos fenómenos de propagación del riesgo no se deben únicamente a las redes.

En este contexto, la idea es demostrar la utilidad de la noción de «transmisión de vulnerabilidad» para dar cuenta de tales efectos de propagación del riesgo a distancia, que pueden transformar un evento limitado y localizado en una catástrofe que abarca un territorio muy extenso. Comenzar por las redes permite una primera lectura de la transmisión de la vulnerabilidad. Se trata también, en este texto, de una tentativa, más allá de la propagación de los riesgos operada por las redes, de descifrar el papel de las relaciones de interdependencias espaciales y funcionales de los objetos y lugares en la propagación de los riesgos y de la vulnerabilidad urbana.

Para hacerlo, comenzaremos por presentar algunos fenómenos de propagación del riesgo, apoyándonos en varios ejemplos de accidentes y catástrofes localizados que acarrearon efectos secundarios más o menos lejanos e imprevisibles. Los ejemplos, algunos de los cuales atañen a los países andinos, permiten no solo demostrar muy concretamente la vulnerabilidad producida por las interdependencias entre redes 
y entre territorios y redes, sino además probar que la propagación del riesgo no se limita al riesgo difundido por las redes.

La segunda parte presenta el funcionamiento sistémico de las ciudades y las múltiples conexiones materiales e inmateriales necesarias para el funcionamiento de un territorio. Esta dimensión estructural de la evolución urbana y territorial permite destacar, en toda generalidad, una problemática de la vulnerabilidad urbana que necesariamente se despliega y propaga $y$, de la suerte, participa plenamente hoy en día en el desarrollo urbano.

En un tercer punto se tratará de interpretar los efectos de propagación y de cadena de fenómenos perjudiciales en términos de transmisión de vulnerabilidad, partiendo de un enfoque del riesgo basado en las nociones de elementos esenciales y de vulnerabilidad. Esta concepción ofrece un marco de formalización de los efectos secundarios en una óptica de transmisión de vulnerabilidad que va más allá de lo que se puede interpretar como la interdependencia de las redes. Esta primera lectura de los «mecanismos de transmisión de vulnerabilidad» se apoya en la hipótesis de una articulación fundamental entre elementos esenciales y territorio.

\section{PROPAGACIÓN DEL RIESGO Y EFECTOS DE CADENA: ALGUNOS EJEMPLOS}

El conocimiento de los fenómenos de propagación de las catástrofes se construye principalmente mediante los análisis de eventos pasados. Están interpretados de manera imprecisa, sea como la difusión del siniestro (Michel-Kerjan, 2003), de la catástrofe (Cartier, 2003) o del riesgo (Longuépée et al., 2009).

\section{1. Riesgos a gran escala}

La tempestad que cayó en el sur de Canadá y el norte de Estados Unidos a inicios de 1998 nos brinda un ejemplo muy estudiado. Este evento excepcional ha dado lugar a algunos análisis detallados de esa experiencia de crisis (Comisión Nicolet, 1999) y al desarrollo de investigaciones sobre los efectos en cascada y nuevas dimensiones del riesgo (Lagadec \& Michel-Kerjan, 2007). La primera consecuencia directa de este evento climático fue la interrupción del abastecimiento eléctrico en el sur de Québec. Se cortaron cuatro de las cinco líneas principales que abastecen a Montreal, $3000 \mathrm{~km}$ de líneas de la red quedaron fuera de servicio y 1400 estaciones repetidoras fueron dañadas o destruidas. En total, más de tres millones de canadienses no contaron con electricidad durante varios días. Esta interrupción del sistema eléctrico tuvo en sí consecuencias directas considerables, pues al privar a los habitantes de esta energía, se cortaron la iluminación y la calefacción en pleno invierno, a $45^{\circ}$ de latitud Norte. El encadenamiento de los efectos debido a la interdependencia de las redes escapó luego a todo control, con un «inicio de hundimiento por placas de las redes vitales de Montreal y de su región» (Lagadec 
\& Michel-Kerjan, 2007): averías en las refinerías, parálisis de los transportes, perturbaciones de las telecomunicaciones, de los bancos, amenaza de pérdida del sistema de abastecimiento de agua... Es este «hundimiento por placas» de partes enteras del funcionamiento social, territorial y económico lo que justifica la noción de «riesgos a gran escala» (Lagadec \& Michel-Kerjan, 2007), demostrando una vulnerabilidad incontrolable vinculada a la complejidad y a la interdependencia de las redes, aunque también a la de los territorios. Este ejemplo extremo muestra una transmisión ininterrumpida de formas de vulnerabilidad que parece desempeñar un papel mayor en la construcción de los riesgos a gran escala. La primera vulnerabilidad es «la exposición al peligro tempestad» de numerosos elementos de la red eléctrica, que fueron destruidos simultáneamente. Otras formas de vulnerabilidad se desplegaron luego hacia otros campos, producidas por «dependencias frente a la red eléctrica vulnerable» o por la «ausencia de alternativas».

El atentado del 11 de septiembre de 2001 en Estados Unidos mostró de igual manera, más allá del traumatismo que representó, que la destrucción de un lugar estratégico puede tener un impacto considerable. En este caso el derrumbe de dos edificios provocó la ruptura de las canalizaciones de agua, que a su vez averió una plataforma técnica del mayor operador de telecomunicaciones del país, lo que tuvo como efecto el corte de centenas de miles de líneas telefónicas y del acceso a Internet, puso fuera de servicio generadores de emergencia, sin contar la interrupción total del tráfico aéreo durante 24 horas en todo el país (Hanley, 2002; Zimmerman, 2002). Al evento le siguió la implantación de un sistema antiterrorista sin precedente, con incidencias a nivel mundial. En este ejemplo preciso lo que se puede destacar es la importancia de los lugares estratégicos desde el punto de vista a la vez funcional y simbólico, cuya destrucción o daño tiene consecuencias en territorios muy extensos y en campos muy diversos. Las redes estuvieron implicadas en la catástrofe (en especial la red telefónica) pero no explican sus repercusiones más importantes. La suspensión del transporte aéreo, traslado de empresas que funcionaban en inmuebles de gran altura o el Patriot Act 1 , son consecuencias directas del atentado que no se propagaron por las redes. Se constituyen así nuevos riesgos en el campo político de las libertades públicas y de las relaciones internacionales. Estas reacciones del mundo social, político y económico revelan y construyen nuevos riesgos, en otros territorios o en otros campos del mundo social.

\section{2. Eventos relativamente menores e impactos considerables}

En las capitales andinas, eventos ciertamente menos dramáticos han mostrado, de otra manera, un encadenamiento de disfunciones que destacan la vulnerabilidad de los territorios2. Fenómenos relativamente menores, que han afectado a objetos y

1 Ley antiterrorista del 26 de octubre de 2001.

2 Véase los estudios de caso de la primera parte este volumen y en especial la síntesis de R. D’Ercole, S. Hardy y J. Robert, pp. 573-591. 
lugares determinados, han tenido un impacto importante en territorios ampliados, mostrando claramente la existencia de elementos y lugares estratégicos, de efectos a escalas contenidas unas en otras, de interdependencia entre redes y de propagación del riesgo. Así, en 2003, la avería de una repetidora diferencial en una central eléctrica, evento puntual y físicamente limitado, provocó un corte de abastecimiento de energía eléctrica en gran parte del Distrito Metropolitano de Quito y de las provincias vecinas. Dado que toda actividad requiere electricidad, este black-out, que duró algunas horas, tuvo como consecuencia la paralización casi total de las actividades en la capital y en una parte del país. La importancia de la estación Santa Rosa, de la que dependía entonces el 70 \% de la electricidad consumida en Quito, explica el considerable impacto de un evento tan circunscrito. Así, este ejemplo no muestra solamente la vulnerabilidad del territorio frente a la red eléctrica, sino que subraya al mismo tiempo la importancia crucial de ciertos lugares y elementos esenciales para el funcionamiento del territorio.

Un ejemplo del mismo tipo constituye la ruptura de una tubería de agua que, en 2008, afectó directamente al abastecimiento de agua potable de gran parte de La Paz3. Los usuarios se vieron privados de agua, lo que afectó a los hogares pero también al sistema de atención médica, a la educación y a la producción económica, por efectos directos o indirectos, algunos de los cuales son a priori poco identificables como el impacto en la economía del ausentismo laboral debido a la suspensión de las clases. Por lo tanto, este efecto lejano del abastecimiento de agua en la economía paceña, que partió de la vulnerabilidad técnica de una canalización esencial, va mucho más allá de la dependencia frente a las redes vitales.

En 2003, la ruptura accidental del oleoducto transecuatoriano contaminó una fuente mayor de abastecimiento de agua para el Distrito Metropolitano de Quito. Este accidente tecnológico comprometió por largo tiempo el aprovisionamiento de agua potable a más de 100000 hogares quiteños y a una cantidad importante de actividades localizadas en la parte norte de la ciudad. Gracias a la existencia de bocatomas suplementarias puestas en servicio algunos años antes para optimizar y regular los caudales de producción y distribución, la empresa metropolitana encargada de la red pudo evitar una interrupción del servicio. Este episodio muestra que un evento perjudicial en un punto clave de una red vital puede ser circunscrito y no afectar sino al manejo técnico interno del sistema de abastecimiento, siempre y cuando se disponga de alternativas de funcionamiento, asimilables a las formas de redundancia bien conocidas por los especialistas en la confiabilidad de los sistemas (Zimmerman, 2002). A imagen del evento engendrado por la tempestad que cayó en el sur de Canadá y el norte de Estados Unidos en 1998 (evocado anteriormente), varias vulnerabilidades habrían podido encadenarse y propagarse para desembocar en efectos de gran amplitud: vulnerabilidad del oleoducto, vulnerabilidad de la red frente al oleoducto, vulnerabilidad de la población, de las actividades económicas y de los servicios públicos por dependencia de la red de agua. La existencia de alternativas detuvo esta cadena.

\footnotetext{
3 Véase en este volumen el artículo de S. Hardy, pp. 545-560.
} 
La caída de ceniza del volcán Pichincha sobre la capital ecuatoriana en 1999 constituyó objetivamente un evento menor desde el estricto punto de vista del peligro. Aun si el territorio afectad o fue muy extenso, el espesor de la capa de ceniza no superó algunos milímetros. Sin embargo, las consecuencias de la reactivación del volcán Pichincha fueron sumamente importantes, tanto por el manejo de esta situación inédita como por el fenómeno físico en sí (Metzger, D’Ercole, Sierra, 1999)4. Mucho más allá del espacio directamente afectado por la caída de ceniza, todo el país se vio afectado en aspectos a la vez técnicos, económicos y políticos. A diferencia de los casos anteriores, la cadena de perturbaciones o daños que afectó el territorio no partió del elemento de una red. Fueron varios elementos esenciales del funcionamiento territorial los concernidos al mismo tiempo. Cada uno de ellos originó un conjunto de efectos secundarios que, a menudo, interactuaron. La paralización del aeropuerto, la desviación del tráfico aéreo, la perturbación por congestionamiento o disfunción de la circulación vial, de las telecomunicaciones y del abastecimiento de agua, el desplazamiento de las personas y de las actividades tuvieron impactos identificables en el plano local y nacional (D’Ercole \& Metzger, 2000). No solo los efectos en cascada descuellan en las redes. Esta experiencia subraya también el papel estratégico muy concreto de ciertas funciones, equipamientos y lugares5.

Los dos eventos que siguen, sin que puedan ser calificados de menores, responden a la misma lógica. Varios elementos esenciales del funcionamiento se ven afectados al mismo tiempo, y solo algunos de ellos corresponden a una lógica de red. En estos casos, sin embargo, los fenómenos destructores presentan una extensión menor que en el ejemplo de Quito y del Pichincha.

La tempestad de granizo que cayó en La Paz en 2002 y las inundaciones y deslizamientos de tierra que provocó, causaron numerosas víctimas en el centro de la ciudad. Este desastre hidroclimático, relativamente localizado al inicio, afectó directamente a ejes de comunicación, al sistema de agua potable, al sistema eléctrico, a edificios públicos y privados, en particular en la zona central. Tales daños directos perturbaron luego de manera importante el funcionamiento de toda la urbe y en particular las operaciones de auxilio6, poniendo en evidencia la vulnerabilidad de La Paz ligada a la de su parte central.

Los efectos de los huaycos que invadieron Lurigancho-Chosica (Lima) en 1987 habrían podido limitarse al distrito en materia de víctimas y de destrucción, si elementos esenciales para el funcionamiento de una parte importante de la aglomeración limeña no hubieran sido dañados: una ruta vial esencial para el abastecimiento de alimentos, una central hidroeléctrica y la bocatoma de la planta de tratamiento de agua potable La Atarjea, principal estación de aprovisionamiento de agua potable de la aglomeración?.

\footnotetext{
4 Véase igualmente el artículo de R. D’Ercole, P. Metzger y A. Sierra en este volumen, pp. 487-499.

5 Véase R. D'Ercole y P. Metzger en este volumen, pp. 893-915.

6 Véase el artículo de S. Hardy en este volumen, pp. 501-514.

7 Véase el artículo de C. Abad en este volumen, pp. 475-486.
} 
De esta suerte, la perturbación o destrucción de objetos o lugares particulares del territorio puede conducir a consecuencias perjudiciales más allá de tales objetos o lugares. Los pocos ejemplos citados, en especial en las ciudades de los países andinos, permiten mostrar la amplitud y diversidad de estos encadenamientos de fenómenos destructores, producto de disfunciones que no necesariamente tienen un origen natural y no corresponden todas a efectos de redes.

\section{CIUDADES, TERRITORIOS Y REDES: ARTICULACIONES GENERADORAS DE VULNERABILIDAD}

\section{1. Interdependencia de los territorios y complejidad}

Los ejemplos anteriores muestran que las modalidades mismas de crecimiento y de organización social, técnica y espacial de la ciudad no solo producen riesgos, sino que los aumentan, los amplifican, los transforman. En toda generalidad, las ciudades y aglomeraciones urbanas pueden definirse y aprehenderse de múltiples maneras. Sin embargo, ciertos términos clave siempre están presentes cuando se quiere dar cuenta de lo que caracteriza a una ciudad: densidad, diversidad, proximidad, interacciones. La ciudad supone una dependencia entre lugares, construida en particular por redes que contribuyen a convertirla en territorios más o menos densos y diversos, espacios continuos y contiguos, en el sentido de que una aglomeración es a la vez un solo territorio y varios. Es el caso en particular de Lima/Callao que constituye un inmenso espacio urbano y, a la par, una yuxtaposición de territorios político-administrativos. Toda ciudad es a la vez un lugar particular del territorio y un territorio particular, definido por múltiples interdependencias funcionales y espaciales, tanto en el seno mismo de la aglomeración como en relación con su entorno.

Múltiples interdependencias entre redes, equipos y servicios nutren el funcionamiento de un territorio. La organización espacial de una ciudad va a reforzar conjuntamente el funcionamiento de un territorio como sistema y la necesidad de las redes. En efecto, según el modelo urbano de la segunda mitad del siglo XX, lo que caracteriza la evolución de las ciudades es la división social del espacio, la disyunción de las funciones urbanas y la multipolaridad. Asociada al desarrollo de la movilidad individual motorizada, tal evolución conduce a la producción de espacios urbanos muy extensos, poco densos, funcionalmente especializados y socialmente segregados. Este tipo de funcionamiento territorial se hace posible por la intensa circulación de personas, bienes, capitales e informaciones entre porciones del territorio, y por importantes intercambios con el entorno, apoyándose todos estos movimientos en una multitud de redes materiales e inmateriales, continuas y discontinuas. Estamos frente a algo del orden del macro sistema, compuesto de objetos que hay que comprender como objetos socio-técnicos, producidos, organizados y regulados por dispositivos técnicos, políticos, sociales, económicos y jurídicos (Callon et al., 2001). 
Para dar cuenta de este tipo de complejidad, a menudo se utiliza el concepto de sistema, que remite a la idea de un conjunto de elementos vinculados que coexisten en una organización o estructura que explica mejor su funcionamiento y su evolución que el solo conjunto de sus componentes (Derycke et al., 1996). Se trata, en este campo teórico, de las nociones de causalidades circulares, bucles o cierres recursivos y retroaccioness entre objetos que componen el sistema y entre el sistema y su entorno, que dan cuenta de la complejidad de su funcionamiento y de su evolución (Levy \& Lussault, 2003). Se puede entonces, a propósito de las ciudades, hablar de sistema territorial complejo, cuyos elementos no son aislables unos de otros. La complejidad de las ciudades (Pigeon, 2007) lleva, en el campo de los riesgos, a características típicas de la complejidad, en particular la no linealidad, la no causalidad o la no proporcionalidad de los fenómenos, todas las cuales participan en la construcción de la vulnerabilidad urbana. Detrás de estas nociones teóricas se alojan muy concretamente mecanismos de propagación del riesgo y de transmisión de vulnerabilidad, difíciles de identificar.

\section{2. Interdependencia de las redes y vulnerabilidad de los territorios}

En un sistema territorial, las redes desempeñan un papel crucial de organización al poner en relación, material e inmaterial, a los elementos que lo componen. Más allá de las grandes infraestructuras que son la red vial, el abastecimiento de agua, el alcantarillado, el aprovisionamiento eléctrico, cuyo peso en la organización y la evolución de las ciudades es bien conocido desde hace tiempo, las redes hard y soft ${ }^{9}$ se multiplican (Egan, 2007). Simultáneamente aumentan también las interconexiones e interdependencias entre redes que, bajo formas diferentes, a partir de infraestructuras lineales o puntuales, visibles o invisibles, cubren el territorio: las vías, el agua, la electricidad, la fibra óptica, las ondas de radio, las comunicaciones, la web... Reconstruir y rastrear estas interdependencias, muy numerosas, no es tarea fácil debido a las discontinuidades materiales, a vínculos muy tenues e indirectos, o a circuitos de dependencia que se cierran en sí mismos. Sin embargo, es necesario identificarlas pues el no funcionamiento de una red tiene inevitablemente consecuencias en otras redes.

Por lo tanto, no existe hoy en día en una gran urbe una red vial o un sistema de abastecimiento de agua potable que pueda prescindir de telecomunicaciones o de electricidad. La producción de energía en sí va a depender del agua, al

8 Estos términos, derivados de la complejidad, hacen referencia a fenómenos que escapan a las normas y a las regularidades, donde las causas y las consecuencias están imbricadas, y cuyos efectos no son proporcionales a las causas.

9 El hard remite a redes clásicas de infraestructura pesada, materiales y muy visibles (la red vial, el agua, el alcantarillado...), aunque también a redes más ligeras que requieren mucho menos infraestructura y son por tanto menos visibles y más inmateriales (telecomunicaciones), mientras que el soft se refiere a las redes interpersonales, virtuales, inmateriales y de cooperación social (Egan, 2007). 
igual que las telecomunicaciones, en especial para los circuitos de enfriamiento. Estas últimas ya no pueden prescindir de satélites, de antenas o de fibra óptica, elementos que por su parte dependen del abastecimiento de energía, subordinado a su vez a la red de telecomunicaciones y al sistema de provisión de agua... Si existe un sistema territorial, su representación no puede sino dibujar una maraña de objetos de toda naturaleza, localizados tanto bajo tierra como en el espacio, en la ciudad o en su entorno, todos vinculados entre sí, no solo por dependencia sino a menudo por interdependencia (Robert et al., 2007). Cada una de estas redes no se resume en su trazado ni en su funcionalidad, en la medida en que va a contribuir a caracterizar los lugares, a definir atributos de esos lugares, al tiempo que va a relacionarlos entre sí (Offner, 2000: 139). En este sentido se puede hablar de una verdadera construcción del territorio por parte de las redes. Simultáneamente, el daño de una red va a afectar necesariamente al territorio y a la población, lo que significa que la vulnerabilidad de las redes nutre la vulnerabilidad del territorio.

De igual manera, las redes están íntimamente ligadas en sus aspectos físicos. A menudo comparten los mismos conductos, transitan por los mismos canales técnicos, situados en particular bajo la red vial. Así, las redes de agua potable, de gas, de electricidad, las fibras ópticas y el alcantarillado, más allá de las conexiones funcionales hoy en día imprescindibles que les permiten cumplir su papel, están relacionadas por una proximidad espacial que refuerza su interdependencia y su vulnerabilidad.

Mientras ciertas redes están identificadas y son elementos clave del funcionamiento urbano, otros elementos, considerados antes como servicios y equipamientos urbanos, funcionan hoy en día igualmente en red o como sistema. Es el caso, por ejemplo, de un «sistema de atención médica» cuando reposa en la implantación de una red de establecimientos de salud y la división del trabajo y del espacio, o del «sistema financiero» cuando consiste en una red de establecimientos y servicios bancarios para la circulación de los flujos financieros y de la información. Paralelamente, estos equipamientos y servicios en red van a depender a su vez, directa e indirectamente, de una multitud de otras redes materiales e inmateriales.

\section{3. Una evolución implícita de la concepción del riesgo}

Todo este panorama del funcionamiento urbano apela a una «geografía de las interdependencias» que destacaría cómo los lugares «hacen sistema» (Offner, 2000: 139). Este modo de funcionamiento territorial representa un potencial de riesgos inimaginable:

«Nuestras sociedades complejas ya no son sino una maraña de nudos, hubs, que concentran poderes colosales de difracción. Efectos masivos de desestructuración son ahora posibles pero están fuera de nuestros paradigmas de referencia» (Lagadec, 2003).

«La maraña de las infraestructuras vitales, de las que somos cada vez más dependientes a escala nacional e internacional, pueden dar a toda 
disfunción local una caja de resonancia inédita o 'desparramar' al nivel local problemas cuya fuente y resolución le escapan» (Miserey, 2006).

Tomando en serio este tipo de problema, la administración Clinton creó en 1996 una comisión de protección de las infraestructuras críticas, definidas como aquellas cuya disfunción o destrucción puede fragilizar la economía o la seguridad nacional de los Estados Unidos. Tales infraestructuras incluyen las telecomunicaciones, las redes de abastecimiento eléctrico, el aprovisionamiento de combustible, el sistema bancario y financiero, los transportes, los sistemas de abastecimiento de agua, los servicios de auxilio y los elementos de la continuidad del gobierno10. Una de las preocupaciones mayores radica en los riesgos ligados a Internet, en la medida en que esta red de circulación de información contribuye directamente a la circulación de capitales, de personas, de mercancías, es decir de cosas a la vez materiales e inmateriales. Esta red multipolar (y sin cabeza) está hoy en día muy imbricada en todas las dimensiones del funcionamiento social. Su alcance no reposa únicamente en elementos físicos y desempeña actualmente un papel mayor en el funcionamiento tanto económico, social y político como físico y material de las sociedades.

La cuestión planteada por las autoridades estadounidenses en cuanto a sus infraestructuras vitales está de cierta manera enmarcada por el paradigma clásico del riesgo, siendo el primer objetivo de esa comisión el inventario y la vigilancia de las amenazas y la vulnerabilidad. Sin embargo, como la posición inicial se refiere a las infraestructuras vitales, es decir elementos esenciales del funcionamiento del territorio, y no a las amenazas, se puede considerar que representa un cuestionamiento implícito del paradigma clásico del riesgo que parte comúnmente de los peligros. Así, en la perspectiva de «riesgos a gran escala», se constata cada vez más que el punto de vista se desplaza. Las acciones de prevención de los riesgos ya no se apoyan en la concepción clásica centrada en las amenazas, lo que significa que la dimensión operacional de evaluación de los riesgos para su prevención no tiene cimiento conceptual.

Al apoyarse en una concepción de los riesgos que parte precisamente de esas «infraestructuras vitales», aunque también de otros elementos esenciales del funcionamiento de un territorio, y en particular de una ciudad, la noción de transmisión de vulnerabilidad adquiere todo su sentido. Se trata de identificar de manera más clara y precisa lo que se propaga cuando se habla de propagación del riesgo, y cuáles son los lugares y los mecanismos que originan tal propagación.

10 Executive Order, EO, 13010, Critical Infrastructure Protection, July 15, 1996. 


\section{LA NOCIÓN DE TRANSMISIÓN DE VULNERABILIDAD}

\section{1. Otro enfoque de los riesgos: los elementos esenciales y su vulnerabilidad}

El enfoque propuesto para seguir la pista de los efectos de propagación y de encadenamiento que se han revelado con ocasión de crisis más o menos importantes, parte de una concepción diferente del riesgo. Esta no se apoya en el paradigma clásico del cruce entre la amenaza y la vulnerabilidad, sino en los elementos esenciales y su vulnerabilidad (D’Ercole \& Metzger, 2002; 2004). Esta manera de concebir el riesgo permite interpretar los efectos de su propagación en medio urbano como la consecuencia de mecanismos de transmisión de vulnerabilidad, algunos conocidos y manejables, otros inciertos e imprevisibles. La hipótesis defendida aquí es que son esos mecanismos de transmisión de la vulnerabilidad, construidos y reforzados por la complejidad de las sociedades modernas, los que construyen el prodigioso potencial de riesgo que ocultan.

Si se considera simplemente que lo que hace el riesgo es la posibilidad de perder aquello a lo que le damos importancia, se va a separar la noción de elemento esencial (enjeu), o lo que está en juego (es decir lo que se puede perder) de la de vulnerabilidad (es decir todas las fragilidades y la ausencia de formas de resiliencia) —D'Ercole \& Metzger, 2009—. En efecto, la concepción tradicional de los riesgos confunde en el mismo término de vulnerabilidad a la vez lo que se puede perder (elementos expuestos), su fragilidad (vulnerabilidad) y la dimensión activa de la vulnerabilidad (es decir la capacidad de engendrar o de modificar las consecuencias de un evento) — véase D'Ercole \& Metzger, 2005-. El punto de vista defendido es que «lo que se puede perder» está en el centro de lo que hace el riesgo y debe por tanto tener un estatus autónomo en la manera de concebir y analizar este último. La filosofía de este razonamiento apuesta a que centrándose en los elementos esenciales de un territorio, y no en la «amenaza», es como se va a poder mejorar la manera en que las sociedades enfrentan las situaciones de riesgo y de crisis.

Los elementos esenciales de un territorio están en parte constituidos por las grandes redes vitales o critical lifelines de las que se habló anteriormente, o por ciertos componentes mayores de tales redes, pero remiten igualmente a objetos del mundo social que no son redes. Definidos como lo imprescindible para el funcionamiento de un territorio, esos elementos esenciales son, más allá de las redes logísticas del funcionamiento urbano, todo lo que es indispensable para la población, la economía y el manejo del territorio11. El artículo «Cómo

11 Como ejemplo, la investigación sobre los elementos esenciales del territorio del Distrito Metropolitano de Quito trató los siguientes campos: población, educación, salud, recreación, patrimonio, cultura, agua, electricidad, abastecimiento alimentario, comunicaciones (transporte y telecomunicaciones), aprovisionamiento de combustible, valor del suelo, capitalidad, empresas, administración (D’Ercole \& Metzger, 2002). 
proteger nuestras grandes redes vitales» (Lagadec \& Michel-Kerjan, 2007), por su título en sí, procede efectivamente de esta filosofía que consiste en interesarse en los elementos esenciales para el funcionamiento de un territorio para luego analizar su vulnerabilidad. En el mismo orden de ideas, la comisión destinada a la protección de las infraestructuras estratégicas en Estados Unidos parte de una concepción del riesgo que se basa en cierta cantidad de elementos identificados como esenciales para el funcionamiento del país. Así se observa que incluso si el estatus conceptual de los elementos esenciales de un territorio no está claro en el enfoque científico del riesgo, tales elementos son efectivamente el punto de partida de procedimientos con objetivos operacionales de prevención, en especial en la perspectiva de los riesgos a gran escala. Este enfoque de la prevención de los riesgos significa colocar los elementos esenciales en el centro de la cuestión, es decir que cuestiona el enfoque clásico cuyo pilar es la amenaza.

Lo que hace la vulnerabilidad de los elementos esenciales se entiende como todas las «posibilidades de perturbar o interrumpir» su funcionamiento. La vulnerabilidad de los elementos esenciales se concibe entonces como la resultante final de diferentes características del elemento esencial y de sus relaciones con el entorno. Algunas de esas características o relaciones van a aumentar la posibilidad de daño o de no funcionamiento del elemento (en particular la exposición a las amenazas) y, por ende, a agravar su vulnerabilidad. Otras hacen referencia a características de resiliencia, en el sentido en que van a acrecentar la capacidad de reacción frente a los accidentes y catástrofes y por tanto disminuir la vulnerabilidad (por ejemplo la existencia de alternativas de funcionamiento). Para detectar lo que hace la vulnerabilidad de un elemento esencial, sea cual fuere, se pueden utilizar criterios basados en seis formas de vulnerabilidad12, entre ellas la dependencia de elementos externos y las alternativas de funcionamiento. Como se observó en los ejemplos presentados, la vulnerabilidad de un elemento esencial por su dependencia frente a otros elementos o sistemas del funcionamiento territorial, o por la ausencia de alternativas para su funcionamiento, es directamente inteligible y permite identificar los caminos de propagación del riesgo más fácilmente detectables. Se trata efectivamente de una transmisión de vulnerabilidad.

\section{2. Mecanismos de transmisión de la vulnerabilidad}

Partiendo de esta concepción del riesgo articulada en las nociones de elemento esencial y devulnerabilidad, se puede plantear la hipótesis de que en los mecanismos de propagación del riesgo, es la vulnerabilidad lo que se transmite, principalmente por las dependencias y la ausencia de alternativas de funcionamiento. Es lo que se puede observar en especial con ocasión de crisis provocadas por efectos dominó

12 En Quito (D’Ercole \& Metzger, 2002) se utilizaron seis criterios de análisis de la vulnerabilidad de los elementos esenciales: la vulnerabilidad «intrínseca»; la exposición de los elementos esenciales a las amenazas de origen natural y antrópico y su susceptibilidad al daño; la dependencia; la capacidad de control; las alternativas de funcionamiento; el nivel de preparación para el manejo de crisis. 
no controlados. En efecto, los casos presentados en la primera parte del artículo mostraron que la vulnerabilidad de una red eléctrica por ejemplo, ya sea con ocasión de una avería técnica o de un evento climático excepcional, puede tener como consecuencia la interrupción o una fuerte perturbación de las redes de agua, de transporte, de provisión de combustible, etc. Se asiste entonces al daño, la perturbación e incluso al no funcionamiento total de elementos esenciales que no hacen parte del sistema eléctrico, por el simple hecho de la vulnerabilidad de la red eléctrica. Considerando que la vulnerabilidad de un elemento esencial comprende todo lo que puede perturbar o interrumpir su funcionamiento, es entonces efectivamente algo del orden de la vulnerabilidad lo que se transmite entre elementos esenciales interdependientes. Simultáneamente, como el territorio depende del abastecimiento eléctrico, se puede considerar que la vulnerabilidad de la red eléctrica hace vulnerable a todo el territorio que depende de ella. Se ve entonces dibujarse un conjunto de mecanismos del orden de la transmisión de la vulnerabilidad que operan en las complejas interrelaciones entre elementos esenciales y entre elementos esenciales y territorio.

Partiendo de estas reflexiones, se pueden identificar a priori cuatro mecanismos de transmisión de vulnerabilidad: del territorio hacia los elementos esenciales; entre elementos esenciales; de los elementos esenciales hacia el territorio; entre territorios. Los más activos son al parecer los tres primeros mecanismos.

\section{3. La transmisión de vulnerabilidad del territorio a los elementos esenciales: por localización}

La vulnerabilidad puede transmitirse del territorio a los elementos esenciales por localización. La noción de «vulnerabilidad espacial» (véase D’Ercole \& Metzger, 2004) da cuenta de la vulnerabilidad que caracteriza a los lugares y que va a transmitirse a los elementos esenciales por el simple hecho de su localización: la exposición a las amenazas, sean estas de origen natural o no, y los problemas de accesibilidad. En efecto, la presencia de amenazas o la limitada accesibilidad de un lugar marca la existencia de una vulnerabilidad a priori del territorio, que necesariamente va a transmitirse, de manera directa o indirecta, a los elementos que este aloja. Por ejemplo, un hospital situado en un lugar caracterizado por frecuentes inundaciones o una mala accesibilidad heredará inevitablemente esas características generales del lugar: estará expuesto a las inundaciones y será poco accesible, lo que lo hará vulnerable. Se puede entonces afirmar que la vulnerabilidad espacial se transmite por conjunción espacial. A falta de análisis particular de los elementos esenciales en sí, la vulnerabilidad espacial permite dar cuenta de ciertas dimensiones de la vulnerabilidad de tales elementos, heredades por el simple hecho de su localización. Se trata de una lectura minimalista de la transmisión de la vulnerabilidad. 


\section{4. Transmisión de vulnerabilidad entre elementos esenciales: por dependencia}

La noción de transmisión de vulnerabilidad entre elementos esenciales expresa la idea de que toda forma de vulnerabilidad de uno de ellos va a transmitirse al elemento que depende de él. Esto equivale a decir que un elemento esencial incorpora necesariamente la vulnerabilidad de todos los elementos de que depende. Cuando un hospital depende de las redes de agua potable, de electricidad o vial para funcionar correctamente, es lógico considerar que la vulnerabilidad de cada una de esas infraestructuras se transmite al hospital y que es incorporada a la de este. Esta dependencia puede ser más o menos marcada e irreductible, rastreada en redes materiales o inmateriales y la vulnerabilidad por dependencia es directamente proporcional a la falta de alternativas de funcionamiento.

La disminución de la vulnerabilidad de un elemento esencial puede entonces contribuir directa o indirectamente a reducir la de otros elementos esenciales. Un elemento esencial puede atenuar o controlar la transmisión de vulnerabilidad proveniente de otros elementos esenciales y disminuir así su propia vulnerabilidad, mediante el desarrollo de sus capacidades de control, de las alternativas de funcionamiento y del nivel de preparación para la crisis, limitando o redistribuyendo su dependencia, al desarrollar su autonomía. Para identificar las transmisiones de vulnerabilidad más importantes, hay que hacer un seguimiento de cerca de los vínculos materiales e inmateriales que unen el funcionamiento de los elementos esenciales, remontar las cadenas de dependencia, en especial —aunque no únicamente- por las grandes redes vitales. El análisis de las situaciones de crisis puede ser muy útil para detectar tales cadenas.

Así, para producir un conocimiento útil para la prevención de este mecanismo de transmisión de vulnerabilidad, hay que detectar y seguir muy de cerca todas las dependencias circulares entre elementos (Robert et al., 2008). Sin embargo, la complejidad de las interdependencias obliga necesariamente a interrumpir el rastreo de los vínculos impidiendo toda evaluación completa de la vulnerabilidad de un elemento esencial, pues uno se encuentra rápidamente en un juego de espejos que reflejan al infinito las interdependencias entre los elementos del sistema territorial.

\section{5. La transmisión de vulnerabilidad de los elementos esenciales hacia el territorio: la noción de vulnerabilidad territorial}

Este mecanismo de transmisión de vulnerabilidad puede considerarse como una extensión del mecanismo anterior en la medida en que la vulnerabilidad de uno o varios elementos esenciales se transmite al territorio directamente o por medio de otros elementos esenciales. Este mecanismo puede ser asociado a la noción de vulnerabilidad territorial que parte del principio de que existen, en el seno de todo sistema territorial, elementos y espacios capaces, en razón a la vez de su 
importancia para el territorio y de su vulnerabilidad, de fragilizarlo. En la medida en que el funcionamiento de un territorio depende del buen funcionamiento de sus elementos esenciales, la perturbación o destrucción de uno de ellos, material y localizado, va a afectar necesariamente a todo el territorio. Para tomar un ejemplo simple, el territorio depende de la red de electricidad para funcionar. La perturbación o destrucción de esta, debido a su vulnerabilidad, va a afectar obligatoriamente a todo el territorio atendido por ella. Los lugares que albergan las infraestructuras clave de esta red son entonces lugares estratégicos generadores de vulnerabilidad para todo el territorio.

La vulnerabilidad de un territorio puede entonces aprehenderse a través de la vulnerabilidad de sus elementos esenciales. Estos están más o menos concentrados y son más o menos vulnerables. Partiendo de estos aspectos puntuales de conocimiento, se pueden localizar, caracterizar y jerarquizar los espacios desde los cuales se genera y se difunde la vulnerabilidad al interior de un territorio ( $D^{\prime}$ Ercole \& Metzger, 2009).

Los mecanismos de transmisión de vulnerabilidad de los elementos esenciales hacia el territorio son por tanto constitutivos de la vulnerabilidad territorial y reposan simultáneamente en la dependencia entre elementos esenciales, en las relaciones entre estos y el territorio y en su localización. En otros términos, mientras más importante es un elemento esencial, mayor es la vulnerabilidad del territorio. La importancia del elemento esencial puede verse de dos maneras: por una parte, el territorio depende en gran medida de ese elemento $y$, por otra, dispone de pocas o de ninguna alternativa en caso de no funcionamiento. Por ejemplo, un territorio que depende de un eje vial esencial para su abastecimiento y no tiene alternativa en caso de corte de esa vía, es muy vulnerable. Por otro lado, mientras mayor es la vulnerabilidad de este elemento esencial del que depende el territorio, en particular por su localización y su exposición a la amenaza, aunque también por su dependencia frente a otros elementos esenciales, mayor es la vulnerabilidad del territorio en sí.

\section{6. La transmisión de vulnerabilidad entre territorios}

Este caso teórico plantea la hipótesis, basada en la interdependencia de los territorios, de que la vulnerabilidad de un territorio puede transmitirse a otros. Es el caso de los lugares y en especial de las ciudades que desempeñan un papel particular para otro territorio, como la capital de un país. En este orden de ideas, la vulnerabilidad del Distrito Metropolitano de Quito, capital de Ecuador, puede transmitirse a todo el territorio ecuatoriano, lo que significa que a la vulnerabilidad del país por numerosas razones se suma la de su capital. Se puede plantear también otra hipótesis, según la cual efectos de localización como la proximidad, la contigüidad, el aislamiento o la distancia pueden intervenir en una forma de transmisión de la vulnerabilidad de un territorio hacia otro. Cabe preguntarse también si la transmisión de vulnerabilidad entre territorios no es simplemente la resultante de la dependencia de elementos esenciales situados en otro territorio. 


\section{7. Transmisión de vulnerabilidad y transferencia de riesgos}

Los mecanismos de transmisión de vulnerabilidad no implican el desplazamiento del riesgo, entendido como su disminución en un lugar y su aumento en otro, sino su difusión, es decir una forma de propagación espacial. Esto significa que no todos los mecanismos que se ubican detrás de los procesos de «traslado» o «transferencia» de riesgos son necesariamente interpretables en el marco de la noción de transmisión de vulnerabilidad. Por ejemplo, obras hidráulicas que van a desplazar zonas inundables no constituyen una transmisión de vulnerabilidad. Tampoco el traslado de una fábrica o de una instalación peligrosa es interpretable en términos de transmisión de vulnerabilidad sino que corresponde a la transferencia de riesgos, es decir al desplazamiento de un elemento esencial y de una vulnerabilidad de un lugar a otro ${ }^{13}$.

La noción de transmisión de vulnerabilidad no implica la idea de reducir la vulnerabilidad de un elemento esencial o de un territorio, trasladándola a otro elemento esencial o territorio. La transmisión de vulnerabilidad significa la propagación de la vulnerabilidad como una forma de contaminación, más allá de su campo inmediato espacial o funcional ${ }^{14}$. Por ejemplo, en el caso de los desechos en Lima15, se tienen dos fenómenos: por una parte el desplazamiento del riesgo ligado a la presencia de desechos mediante el traslado de estos de un barrio a otro. Aquí se podría hablar de «transferencia» de vulnerabilidad en la medida en que se reduce la vulnerabilidad de un espacio (los barrios ricos) para acrecentar la de otros lugares (barrios pobres). En este caso la vulnerabilidad no es transmitida, sino desplazada, transferida, externalizada. Supone una acción deliberada. La vulnerabilidad de un espacio debida a la presencia de desechos, en cambio, se va a transmitir a la población que habita o trabaja en la cercanía, por el simple hecho de su proximidad espacial. En otros términos, los espacios concernidos se encuentran expuestos a una amenaza y esta vulnerabilidad va a transmitirse según los mecanismos de transmisión de vulnerabilidad del territorio a los elementos esenciales (vulnerabilidad espacial).

\section{CONCLUSIÓN}

Muchos análisis recientes concuerdan en que las crisis mayores que sacuden regularmente a los territorios no son accidentes en el sentido de eventos coyunturales, sino fenómenos estructurales. En otros términos, son las modalidades mismas de producción y de funcionamiento de las sociedades y los territorios las

\footnotetext{
13 Es por ejemplo el caso en Quito del desplazamiento de una parte de las cisternas de gas (GLP) de El Beaterio a Itulcachi (véase el artículo de J. Estacio, pp. 683-707, en este volumen).

14 Se puede considerar también que la vulnerabilidad se transmite a la manera de un virus informático.

15 Véase el artículo de M. Durand y P. Metzger en este volumen, pp. 623-646.
} 
que, al reforzar la complejidad y las interdependencias, producen situaciones de riesgo que se transforman en crisis.

En este contexto, la noción de «transmisión de vulnerabilidad» es esencial para comprender la globalidad y la complejidad del riesgo que corre un territorio. Adquiere todo su sentido en el marco de los riesgos a gran escala y del análisis de los efectos de propagación, más allá de lo que se puede detectar siguiendo la maraña funcional y espacial de las grandes redes vitales. Trata de dar cuenta, a la vez en el campo conceptual de los riesgos y en aquel, muy concreto, de las catástrofes, de los mecanismos que operan detrás de la vulnerabilidad invisible y otros efectos en cadena que se revelan con ocasión de las situaciones de crisis. Se inscribe en una concepción de los riesgos basada en dos nociones principales: los elementos esenciales de un territorio y la vulnerabilidad. La vulnerabilidad de un territorio reposa fundamentalmente en tres mecanismos de transmisión, esenciales para comprender el riesgo: la vulnerabilidad se transmite entre elementos esenciales por dependencia; la vulnerabilidad de un elemento esencial se transmite a todo el territorio por dependencia y ausencia de alternativas; la vulnerabilidad espacial se transmite a los elementos esenciales por localización. Se puede considerar un cuarto mecanismo, la vulnerabilidad que se transmite de un territorio a otro, pero en este caso se trata de un mecanismo aún poco formalizado.

La idea de transmisión de vulnerabilidad permite señalar una característica de la vulnerabilidad urbana: nunca se limita a un lugar, sino que siempre es capaz de propagarse, de provocar efectos en cadena de tal complejidad que es difícilmente identificable y manejable, e incluso totalmente incontrolable. Los mecanismos de transmisión de vulnerabilidad son portadores de riesgos a gran escala. El desafío es entonces identificarlos y localizar su punto de partida. Es lo que se puede intentar a partir de la noción de vulnerabilidad territorial.

Se puede pues plantear la hipótesis, que queda por verificar, de que la vulnerabilidad no es un atributo estabilizado e impermeable de los lugares, de las cosas y de las sociedades, sino un fenómeno que va a desplegarse y expandirse mediante múltiples interdependencias entre objetos, entre territorios, entre objetos y territorios. Al propagarse de un objeto a otro, va a transformarse, a cambiar de forma y de estado. Dada la complejidad del sistema territorial, hablar de vulnerabilidad de tal o cual lugar o elemento esencial en relación con una amenaza, sísmica o de inundación por ejemplo, es muy reductor, e incluso contraproducente, puesto que la multiplicidad y la complejidad de las interdependencias propagan instantáneamente toda vulnerabilidad en dimensiones difícilmente controlables. Cada conexión entre lugares, entre elementos esenciales, significa una puerta de penetración de la vulnerabilidad de los lugares y de los objetos conectados, cada dependencia significa la apertura a la vulnerabilidad de aquello de lo que se depende.

La transmisión de vulnerabilidad es un mecanismo que da cuenta de la propagación del riesgo entre los objetos del mundo. Implica conjuntamente lo natural y lo social, lo material y lo inmaterial, lo físico y lo institucional, en un proceso general de puesta en peligro de los territorios que toma la vía de todas las interconexiones 
y circuitos del mundo moderno. Es por ello que el análisis de los mecanismos de transmisión de vulnerabilidad es necesario, con la idea de detectar los objetos, los procedimientos y los lugares que permitirían interrumpir esta transmisión.

\section{Referencias citadas}

BECK, U., 2001 - La société du risque, 521 pp.; París: Flammarion.

CALLON, M., LASCOUMES, P. \& BARTHE, Y., 2001 - Agir dans un monde incertain, Essai sur la démocratie tecnique, 358 pp.; París: Le Seuil.

CARTIER, S., 2003 - Réorganisation des réseaux techniques et sociaux : de la crise aux décisions. In: International Conference on Risk, vulnerability and Reliability in Construction, 7 pp.

http://www-lgit.obs.ujf-grenoble.fr/scartier/.../cartier2003conferencealgier.pdf

COMMISSION NICOLET, 1999 - Rapport de la Commission scientifique et technique sur la crise du verglas de janvier 1998, cinq volumes; Montréal: Publications du Québec.

D'ERCOLE, R. \& METZGER, P., 2000 - La vulnérabilité de Quito (Équateur) face à l'activité du Guagua Pichincha. Les premières leçons d'une crise volcanique durable. Cahiers Savoisiens de Géographie, n. ${ }^{\circ}$ 1: 39-52; Université de Savoie.

D'ERCOLE, R. \& METZGER, P., 2002 - Los lugares esenciales del Distrito Metropolitano de Quito, 226 pp.; Quito, Ecuador: MDMQ, IRD. Colección Quito Metropolitano. http://www.savgis.org/estudios-realizados.html

D'ERCOLE, R. \& METZGER, P., 2004 - Vulnerabilidad del Distrito Metropolitano de Quito, 496 pp.; Quito, Ecuador: MDMQ, IRD. Colección Quito Metropolitano. http://www.savgis.org/estudios-realizados.html

D'ERCOLE, R. \& METZGER, P., 2005 - Repenser le concept de risque pour une gestion préventive du territoire. Pangea, $\mathbf{n} .^{\circ} \mathbf{4 3 - 4 4}$, junio-diciembre: 19-36.

D'ERCOLE, R. \& METZGER, P., 2009 - La vulnérabilité territoriale : une nouvelle approche des risques en milieu urbain. Cybergeo: European Journal of Geography [En línea], Dossiers, Vulnérabilités urbaines au sud, document 447, colocado en internet el 31 de marzo de 2009.

http://www.cybergeo.eu/index22022.html

DERYCKE, P.-H., HURIOT, J.-M. \& PUMAIN, D., 1996 - Penser la ville. Théories et modèles, 335 pp.; París: Anthropos. Collection Villes.

EGAN, M. J., 2007 - Anticipating Future Vulnerability: Defining Characteristics of Increasing Critical Infrastructure-like systems. Journal of Contingencies and Crisis Management, vol 15 (1), marzo: 4-17.

GODARD, O., HENRY, C., LAGADEC, P. \& MICHEL-KERJAN, E., 2002 - Traité des nouveaux risques, 620 pp.; París: Gallimard.

HANLEY, R., 2002 - Développement durable, réseaux techniques et terrorisme. Métropolis, Flux, 2002/1, $\mathbf{n} .^{\circ}$ 47: 80-83.

LAGADEC, P., 2003 - Risques, crise et gouvernance : ruptures d'horizons, rupture de paradigme. Réalités industrielles, mai: 5-11.

LAGADEC, P. \& MICHEL-KERJAN, E., 2007 - Comment protéger nos grands réseaux vitaux. Les dossiers de La Recherche, $\mathbf{n}^{\circ} \mathbf{2 6}$, febrero-abril: 38-42.

LEVY, J. \& LUSSAULT, M., 2003 - Dictionnaire de la géographie, de l'espace et des sociétés, 1033 pp.; París: Belin. 
LONGUÉPÉE, J., CALLENS, S. \& DUEZ, P., 2008 - Développement durable et territoires, Dossier 11 : Catastrophes et Territoires. Introduction au dossier $n^{\circ} 11$ Catastrophes et Territoires. http://developpementdurable.revues.org/index6763.html

METZGER, P., D'ERCOLE, R. \& SIERRA, A., 1999 - Political and scientific uncertainties in volcanic risk management: The yellow alert in Quito in October 1998. Geojournal, 49: 213-221.

MICHEL-KERJAN, E., 1999 - Risques à grande échelle dans les systèmes en réseau : quelques interrogations. Cahier du Laboratoire d'Econométrie de l'École Polytechnique, n. ${ }^{\circ}$ 505: 25 pp.; París.

MICHEL-KERJAN, E., 2003 - Risques catastrophiques et réseaux vitaux : de nouvelles vulnérabilités. Flux, n. ${ }^{\circ}$ 51: 6-15.

MISEREY, Y., 2006 - Nous sommes passés de l'accident au chaos. Le Figaro Economie, 6, noviembre.

MORIN, E., 1990 - Introduction à la pensée complexe, 158 pp.; París: Seuil.

OFFNER, J.M., 2000 - Réseaux et dynamique urbaine. In: La ville et l'urbain, l'état des savoirs, (T. Paquot, M. Lussault \& S. Body-Gendrot, eds.): 137-145; París: La découverte.

PIGEON, P., 2007 - L'environnement au défi de I'urbanisation, 189 pp.; Rennes: Presses Universitaires de Rennes.

ROBERT, B., BROUSSEAU, D. \& SABOURIN, J.-P., 2008 - Interdépendances et services essentiels, un défi relevé à Montréal et à Québec; Québec: Colloque 2008 sur la Sécurité Civile. www.msp.gouv.ca/secivile/

ZIMMERMAN, R., 2002 - Enjeux et gestion des interactions entre les différents réseaux d'infrastructure. Flux, 2002/1, n. ${ }^{\circ}$ 47: 54-68. 